\author{
Justyna Kłopotowska \\ ORCID: 0000-0001-6452-504X \\ Universität Szczecin, Szczecin
}

DOI: $10.19195 / 0435-5865.143 .4$

\title{
Die sexuellen Neurosen unserer Eltern von Lukas Bärfuss und die Frage nach der Tabuisierung von Sexualität
}

\begin{abstract}
s
Sexualität der Menschen, die als eines von vielen Themen im Alltag erscheint und die auch literarisch aus vielerlei Perspektiven aufgegriffen wird, wird hier und da gerne tabuisiert. Lukas Bärfuss, Vertreter der jungen, deutschsprachigen Schweizer Literatur, setzt sich mit diesem Thema auf eine sehr provozierende Art und Weise auseinander. In seinem Theatertext Die sexuellen Neurosen unserer Eltern führt er die Leser in ein verklemmtes Milieu, in dem seine geistig behinderte Protagonistin Dora ihre Sexualität entdeckt. Was daraus wird, wie der Autor damit umgeht und es in Sprache umsetzt, wird zum Thema der Überlegungen.
\end{abstract}

Schlüsselwörter: Schweizer Literatur, Bärfuss, Tabu, Sexualität

\section{The sexual neuroses of our parents by Lukas Bärfuss and the question of the tabooing of sexuality}

People's sexuality, which appears as one of many topics in everyday life and which has been approached in the literature from a variety of perspectives, has often been tabooed. Lukas Bärfuss, a representative of the young Swiss German literature, is dealing with this topic in a very provocative way. In his theatrical text The sexual neuroses of our parents, he leads the readers into an inhibited milieu in which his mentally handicapped protagonist Dora discovers her sexuality. What becomes of it, how the author deals with it and puts it to practice, will become the subject of the considerations.

Keywords: Swiss German literature, Bärfuss, tabu, sexuality

Justyna Kłopotowska, Uniwersytet Szczeciński, Instytut Filologii Germańskiej, Al. Piastów 40b, 71-065 Szczecin, Polen, E-Mail: justyna.klopotowska@usz.edu.pl

Received: 12.09.2017, accepted: 4.05.2018

Germanica Wratislaviensia 143, 2018

(C) for this edition by CNS 
Neurose als Zustand allzu großer Aufregung und Reizbarkeit, die vielerlei Ängste und unkontrolliertes Benehmen hervorrufen kann, u.a. Zwangsstörungen, Hypochondrien und Hysterien, kann einem Texttitel eher schwer fallen. Ebenso schwer scheint es zu sein, dass ein Text mit solch einem Titel, also Die sexuellen Neurosen unserer Eltern, flink und unbeschwert geschrieben werden kann. Eher würde ein Werk, das so hieße, auf den ersten Blick trüb und abschreckend wirken. Aber! Nachdem man die Arbeit von Lukas Bärfuss gelesen hat, erweisen sich all die ersten Eindrücke sehr schnell als gelungene Provokation, was den potenziellen Leser desto mehr anlocken sollte, zumal sich hinter den erwähnten Neurosen eine sehr interessante, zugleich aber auch heikle Geschichte versteckt. Eine Geschichte, die Fragen nach Sexualität behinderter Menschen aufgreift und sie den Tabus, Klischees und Vorurteilen gegenüberstellt, welche Menschen besonders in kleinen Milieus beherrschen. $\mathrm{Zu}$ untersuchen sind dabei Konstellationen, die in diesem Zusammenhang im Primärtext entstehen, sowie die Einordnung des Werkes in die aktuelle Dramenlandschaft.

\section{Zum Autor}

Bärfuss, der Provokateur, der sich an solch einen Text mit solch einem Titel gewagt und ihn mit der Sexualität verknüpft hat, gehört heutzutage zu den bedeutendsten und einflussreichsten, deutschsprachigen Schriftstellern und Dramatikern, die in den 1990er Jahren zu schreiben begannen. Er vertritt die neueste Generation der in der Schweiz schreibenden Autoren, die ihr Schaffen vielmehr global und weltoffen zu verstehen vermögen, was für die Helvetia um die Jahrtausendwende ebenso paradigmatisch zu sein scheint. Das Postmillenium, das diese Generation prägt und das nach Reto Sorg mit der ,neuen Entspanntheit" verbunden ist (Weber, Burkard: 2-7) - was heißt, dass die Literatur nach 2000 der vorangehenden Epoche nicht mehr oppositionell steht, sondern nur auf viele formelle Experimente verzichtet - ermöglicht, direkt und unverhüllt Themen anzuschneiden, die dabei globale Probleme als Ausgangspunkt jeder Auseinandersetzung möglich machen.

Der heute 46 Jahre alte Schweizer Autor ist in seinem literarischen Werdegang von vielerlei Erfahrungen und Erlebnissen geprägt worden. Bevor er zu schreiben anfing, arbeitete er u.a. als Tabakbauer, Eisenleger oder Gärtner. ${ }^{1}$ Er schreibt sowohl Prosa als auch Dramen, wobei das zweite in seinem Euvre überwiegend erscheint. 1998 hat er zusammen mit Samuel Schwarz die Gruppe 400asa gegründet (der Ausdruck stammt aus der Sprache der Photographie und ist ein Mass für die Lichtempfindlichkeit von Filmen), 1999 erschien ihr modernes Manifest Bekenntnis 99, in dem sie auf das Textbuch als einziges Requisit im Theater ver-

${ }^{1}$ Vgl. http://www.munzinger.de/search/go/document.jsp?id=00000025289 (Zugriff am 14.07.2015). 
wiesen. ${ }^{2}$ Die Theaterkonzeption von 400asa rückt die Herleitung ideeller Parolen ins Zentrum:

Was im dänischen Kino funktioniert, funktioniert auch im deutschsprachigen Theater! Es lebe die Beschränkung der künstlerischen Mittel! Es lebe der Asketismus! Schluss mit manieristischen Bühnenbildern! Schluss mit selbstzweckhaft eingesetzten Videolein- und Plexiglaswänden, die dazu führen, dass eine Produktion mit einem Budget unter 100000 Dollar nicht mehr ernst genommen wird. (www.400asa.ch.)

Die Begrenzung des Theaters aufs Nötigste wurde zudem durch die Idee der Kunstbefreiung im globalen Sinne ergänzt und gefördert:

Keine Festung Schweiz! Keine Festung Europa! Überhaupt keine Festungen! Wir verhandeln nicht! Die Kunst verhandelt nicht! Keine Ansprüche an Künstler! Sie schulden der Gesellschaft nichts, die Gesellschaft ihnen alles! Wer Ansprüche hat, soll sie selbst verwirklichen! Befreit die Kunst! (ebd.)

$\mathrm{Zu}$ den berühmtesten Bühnenstücken von Bärfuss gehören außer Den sexuellen Neurosen unserer Eltern (2003), Der Bus (2005), Alices Reise in die Schweiz (2005), Öl (2009) oder Zwanzigtausend Seiten (2012), zu seinen Prosawerken Die toten Männer (2002), Hundert Tage (2008), Koala (2014) und seine Essays Stil und Moral (2015). Für sein Schaffen wurde er mit mehreren Preisen ausgezeichnet, u.a. dem Anna Seghers-Preis (2008), dem Solothurner Literaturpreis (2014), dem Schweizer Buchpreis (2014) oder auch dem Johann-Peter-Hebel-Preis (2016).

In den Texten und Stücken des Schweizer Autors tauchen überall provokante Themen auf. Man liest über blinde Religiosität, gesellschaftliche Verwerfungen und Klassenablehnungen, alle möglichen Klischees, Radikalismen und Fundamentalismen, mentale Enge, Verzahnung in Konventionen und Gedankensprüngen, aber auch über das Verdrängen aus dem Bewusstsein von Grundinstinkten, die mit den Naturgesetzen im Einklang stehen. Bärfuss, der gerne unangenehme und verpönte Themen anspricht, um allgemein geltende Regeln und Normen zu hinterfragen und zugleich Tabus brechen zu können, hat keine Angst vor Herausforderungen und ungewollten Themen, wodurch er auch großes Aufsehen in anderen, nicht nur europäischen, Ländern erregt, von Kritikern entweder verhasst oder verhätschelt als Schöpfer „ungewöhnlicher Bilder mit besonderer, sprachlicher Richtigkeit" (Gespräch mit Lukas Bärfuss: http://www.teatr-pismo.pl/ludzie/650/ filozof_i_poeta._rozmowa_z_lukasem_bärfussem). Auch selber äußert er sich zu seinem Schaffen. Man liest u.a.:

Meine Gestalten sind vielleicht untypisch, aber sie stehen im Zentrum der Ereignisse und erleben das, was uns allen nah ist, wobei diese Ereignisse für uns keine Gefahr bedeuten, weil wir meistens Unterstützung in gesellschaftlichen Strukturen haben: in der Arbeit, zu Hause. Die meisten von uns besitzen eine Rolle, die vor solchen Erfahrungen schützt. Und mich interessiert vor allem das, was unabwendbar ist. Dies hängt auch mit der Dramaturgie zusammen;

${ }^{2}$ Vgl. http://400asa.ch/400asa/geschichte.php (Zugriff am 14.07.2015), http://tls.theaterwissenschaft.ch/wiki/Lukas_Bärfuss (Zugriff am 14.07.2015) oder http://www.400asa.ch/theater/archiv/itnacht/bekenntnis99.pdf (Zugriff am 14.07.2015). 
Geschichten, die im Theater erzählt werden, beschäftigen sich oft mit Menschen, die von der Allgemeinheit abgestoßen, eine besondere Weise der Weltanschauung entwickeln, wodurch sie auf bestimmte Sachen so hinweisen. Wie ist die Welt, in der wir leben, wie funktioniert sie und mit welchen Gesetzen, warum gelten eben solche und nicht andere Gesetze und worauf beruht genau die conditio humana? Auf welche Art und Weise kommen wir mit ihr in Berührung und auf welche Art und Weise kommt sie mit uns in Berührung? Meine Gestalten helfen mir diese Fragen erforschen. Die Schriftstellerei ist für mich ein Versuch, sie zu erklären. Deswegen finde ich selber, dass ich im vergleichbaren Maße Philosoph sowie Poet bin. (ebd.)

Seine Gestalten sprechen in ihren Geschichten/Schicksalen und Erlebnissen Aspekte an, die sich also ständig im Offenen und Kritischen hin und her bewegen. Bärfuss empfindet Leidenschaft für schwierige Situationen, die er aufzunehmen und zu versprachlichen versucht. Sexualität ist bei ihm demnach eines von vielen Themen, das mit seinem sozialen Engagement im Einklang steht und das aus dem Lebensprozess geholt wird. Sie wird in dem von Bärfuss genannten Untypischen, aber nicht nur mit einer allgemein geltenden Tabuisierung verbunden, sondern mit Behinderungen und Behinderten, was die Problematik desto deutlicher zu hinterfragen erlaubt und die ,für verlässlich gehaltene[n] Moralsysteme erschüttert“ (http://www.literaturfestival.com/archiv/teilnehmer/autoren/2014/ lukas-baerfuss). Zwar meint der Autor, er vermeide jeden autobiographischen Einfluss in seiner Literatur, aber er hebt zugleich hervor, er gehe bei der Stoffsuche ständig von seiner Person aus, die politisch und sozial sehr stark geprägt worden ist. Die Texte, die er schreibt, bewegen auch aus diesem Grund. Er meint: „Ich komme aus komplizierten Verhältnissen. In der Patchworkfamilie, in der ich aufgewachsen bin, spiegeln sich die großen Sehnsüchte und Verwerfungen der Zeitgeschichte. Deswegen wohl ist meine Person vom Politischen nicht leicht zu trennen“ (Cavelty).

Der Scharfsinn, die Empfindlichkeit gegen Ungerechtigkeit und zugleich das Verständnis für die menschlichen Abgründe machen den Hintergrund aus, mit dem ohnehin das ganze Schaffen von Bärfuss - nicht nur seine Theatertexte - zu betrachten ist.

\section{Zum Werk}

Dora, die Protagonistin des Bärfusschen Werkes, ist geistig gestört. Sie wohnt mit ihren Eltern in einem verklemmten Milieu, wo sie sich jahrelang unter dem Einfluss von starken Psychopharmaka befindet, weil die Eltern behaupten, dass es besser und sicherer für die Gemeinschaft sei sowie für Dora selber, die ohne Tabletten wild und unkontrolliert ihre Gefühle äußert.

Die Ausgangssituation etabliert eine Zwiespältigkeit. Die Mutter merkt, nachdem Doras alter Arzt gestorben ist, dass ihre Tochter nach der Verabreichung von so vielen Tabletten gar nichts empfindet. Sie sagt besorgt zu dem neuen Arzt: 
[...] Sie schreit nicht mehr, aber sie lacht auch kaum, weint nie, ißt, was man ihr vorsetzt. Seit zwei Jahren hat sie kein rechtes Gespräch geführt, nur Phrasen wiederholt sie, Aufgeschnapptes. Hin und wieder summt sie ein Lied, von dem keiner weiß, wo sie es herhat. [...] Manchmal wünsche ich die Tobsuchtsanfälle zurück. Doras Lachen, das lauter war als das Lachen meines Mannes. Man glaubte, in meinem Mädchen hocke ein Seemann oder ein Schlachter. (Bärfuss 2015: 73)

Und obwohl ihr der Arzt davon abrät, entscheidet sie sich dafür, die Medikamente abzusetzen. ${ }^{3}$ Dora, die von nun an keine Arzneien mehr einnimmt, beginnt das Leben zu entdecken. Die Mutter ist glücklich. ${ }^{4}$ Zwar kann die Protagonistin ihr Leben nicht unabhängig gestalten (sie geht immer mit einem Band am Hals, wo ein Zettel mit ihrem Namen und ihrer Adresse hängen bleibt, falls sie sich verlaufen würde), kann es aber beobachten und genießen - auf eine ihr spezifische Art und Weise. In dem Gemüseladen, wo sie seit Jahren als Aushilfe arbeitet, lernt sie einen Parfümvertreter kennen (der als „,einer Herr“ genannt wird), auf den sie sich verlässt, der sie mit ins Hotel nimmt, wo er sie verprügelt und mit ihr Sex hat. Als sie mit blauen Flecken nach Hause zurückkommt, sind die Eltern verblüfft. ${ }^{5}$ Dora meint aber, sie fühle sich gut und finde es nicht schlecht, sondern eher angenehm, was der Mann mit ihr getan hat. Die Mutter geht mit ihr zum Arzt, der sie untersucht und sexuell aufklärt (er spricht u.a. vom Zivil- und Strafrecht, verschreibt ihr Pillen und verweist darauf, dass sie Recht darauf hat, ihre eigene Moral in der Liebe zu haben - wenn es überhaupt solch eine geben darf). Zwar verspricht Dora der Mutter, mit dem Mann nie wieder hinzugehen, aber nachdem sie ihre sexuellen Lüste entdeckt hat, will sie es nicht aufgeben, begehrt den Mann immer mehr und trifft sich mit ihm daher regelmäßig. ${ }^{6}$ Sie empfindet Spaß am harten Sex, Verprügelungen und Beleidigungen, die ihr seitens des Parfümvertreters zuteil werden. ${ }^{7}$ Als es sich herausstellt, dass sie schwanger ist, will die Mutter sofort eingreifen und das Kind wegmachen. Dora wird dazu überredet und lässt die Abreibung zu. ${ }^{8}$ Außerdem werden ihre Eileiter unterbunden. Als „der feine Herr“, der zunächst

${ }^{3}$ Sie sagt: „Zum letzten Mal hatte ich meine Tochter, als sie ein Kind war. Jetzt ist sie beinahe erwachsen. Ich sah, wie sich ihr Körper veränderte. Ich möchte sehen, wie verändert ihr Inneres ist. Was unter dem ewig gleichen Gesicht liegt“" (Bärfuss 2015: 74).

${ }^{4}$ Sie sagt zu dem Arzt: „Es war die richtige Entscheidung. Sie ist wieder ein Mensch. Wenn ich die letzten Jahre überblicke, befällt mich ein schlechtes Gewissen, und ich werde wütend auf mich selbst" (Bärfuss 2015: 82).

${ }^{5}$ Die Mutter sagt: „Meine Kleine, überall blaue Flecken hat meine Kleine. [...] Du verstehst nicht, was er dir angetan hat. Sogar das Geld hat er dir genommen, dieser böse Mensch. [...] Verdammter Dreckskerl“" (Bärfuss 2015: 86).

${ }^{6}$ Sie sagt zu ,dem feinen Herrn“ am Bahnhof beim nächsten Treffen, als er sie nicht erkennen will, u.a.: „Nicht wieder wegschicken. Nehmen Sie mich mit. Ich will einmal länger ficken als nur eine halbe Stunde“ (Bärfuss 2015: 93).

${ }^{7}$ Er sagt, sie stinke wie Ochsenscheiße: „Du riechst nicht, du stinkst. Du stinkst erbärmlich“ (Bärfuss: 95).

${ }^{8}$ Sie sagt danach: ,wie der Doktor mit dem Schlauch gekommen ist, habe ich gedacht, es wird gefickt. Aber dann hat es nicht gestoßen, sondern gesaugt. Auch nicht schlecht. Und es hat geklungen, wie wenn man mit einem Strohhalm das Glas leerschlürft" (Bärfuss 2015: 104). 
behauptet, er könne mit ihr sogar eine Familie gründen, ${ }^{9}$ dies erfährt, verlässt er sie. Er gibt ihr die 10 Franken zurück, die er ihr am Anfang genommen hat und sagt, sie seien jetzt quitt - als wäre sie für ihn in diesem Moment nur noch eine Prostituierte. Am Ende schickt er sie zum Bahnhof, wo sie den Zug nach Russland nehmen sollte, wohin auch „der feine Herr" ihr folgen würde - der kleinen Russin, wie er sie mal genannt hat, die ,ein wenig von jener letzten Zarentochter [hat], verarmt, entehrt, gestrandet zwischen Kartoffeln und Suppengemüse" (Bärfuss 2015: 84). Dora, die seiner wirklichen Einstellung völlig unbewusst bleibt, freut sich aufs Wiedersehen.

Wenn Mama das jetzt sehen könnte. Sie hat behauptet, ich würde das Geld nie wieder sehen. Und daß wir nach Rußland fahren, hat sie auch nicht geglaubt und wollte mich gar nicht gehen lassen. Und Papa hat gesagt, einmal mußte dieser Zeitpunkt ja kommen, aber Mama hat geweint, und dann hat er gesagt, wer loßlässt, hat die Hände frei, aber Mama hat geweint, und Papa hat gesagt, wir haben sie gut vorbereitet, und Mama hat meinen Koffer gepackt und hat geweint und hat auch nicht aufgehört, als ich ihr versprochen habe, aus Rußand eine Ansichtskarte zu schicken. (Bärfuss 2015: 127)

Der Text, der in 35 Teile gegliedert wird, scheint die Tabuisierung der Sexualität schlagkräftig zu schildern. Der Autor zeigt den Weg ins Bewusstsein und das Heranwachsen der eigenen Sexualität bei Dora, einer komplexen Figur, die mal als naives Kind und mal als selbstbewusste Frau mit starkem Sexualtrieb daherkommt. Julian Ingelmann schreibt hierzu: „Ihre Weltsicht changiert zwischen brutaler Ehrlichkeit, trockener Analyse und totaler Ahnungslosigkeit" (Ingelmann). Die im Text verbreitete Behauptung, Sex sei vor allem etwas Privates, wovon man nicht sprechen sollte, ist hier in den Klischees und verschlossenen Verhaltensweisen zu sehen, vor allem bei den Eltern (die Doras Fragen nach ihrer Sexualität unverschämt finden ${ }^{10}$ ), aber auch bei dem Klein-Leute-Milieu, zum Beispiel bei dem Chef des Gemüseladens, der - von Dora angemacht und geküsst - statt mit ihr darüber zumindest zu reden, ihr eine Ohrfeige verpasst und sie sofort entlässt. Es gibt dabei keinen Willen zur Aufklärung und zum offenen Gespräch über die Sexualität, die Dora bei sich entdeckt. Das, was es gibt, ist die Doppelmoral des Milieus, das einerseits - wie die Eltern - nach höheren Werten zu streben glaubt, das aber auch andererseits in diesem vermeintlichen Glauben daran eine Abtrei-

${ }^{9}$ Er sagt: „Das wäre was geworden, du und ich und ein Kind. Eine hübsche Familie [...]“ (Bärfuss 2015: 108).

${ }^{10} \mathrm{Vgl}$. folgenden Dialog:

DORA: Warum hast du es mir nicht erzählt.

MUTTER: Was habe ich dir nicht erzählt.

DORA: Daß du fickst.

MUTTER: Weil es dich nicht angeht.

DORA: In meinem ganzen Leben habe ich noch nie so etwas Schönes gesehen. Du sahst aus wie ein richtiger Engel.

MUTTER: Sei still.

(Bärfuss 2015: 116). 
bung durchsetzt, weil es nicht akzeptiert, dass auch Behinderte Sex, Familien, Beziehungen und Verhältnisse haben können: „Ihr [Doras - J.K.] Charakter ist als tragikomischer Gegenpol zur spießbürgerlichen Pseudoidylle ihrer Eltern konzipiert.“ (Ingelmann: ebd). Der einzige, der dem Milieu entgegengestellt wird, ist der Arzt, der Doras Sexualverhalten nicht aburteilt. ${ }^{11}$ Er sagt u.a.:

Es ist nichts Schlechtes, wirklich nicht, laß dir das nicht einreden. Der Mensch entsteht daraus, jeder, stell dir vor, jeder auf dieselbe Weise, mit und durch dieses Wunder. Und darum ist es gut, hast du es für dich entdeckt. Auch du sollst es erleben dürfen, ja, obwohl man es euch früher verboten hat. Lange genug hat man nicht wahrhaben wollen, dass ihr eine Sexualität habt. Aber heute sehen wir das neu und anders. Es ist nicht nur dazu da, nicht nur fürs Kinderkriegen, bestimmt nicht, obwohl noch manche Leute das vertreten. (Bärfuss 2015: 88)

Besonders brisant ist Doras Beziehung zu „dem feinen Herrn“. Man erfährt nicht direkt, wortwörtlich, ob er „Doras Unwissenheit schamlos aus[nutzt], um seine eigenen Triebe zu befriedigen“ (Ingelmann: ebd.) oder ob das Mädchen wirklich vergewaltigt wird. Eher stellt man sich hier die Frage, ob er ,nur die etwas unkonventionellen Wünsche einer sexuell unerfahrenen Jugendlichen [erfüllt].“ (ebd). Leider kriegt man da keine Antwort von dem Autor. Stattdessen „erschöpft er sich [lieber] in Andeutungen.“ (ebd.).

Die Beschreibung des sexuellen Verhaltens von Dora erfolgt schier naturalistisch. Ihre Aussagen sind unverhüllt und unverschämt, ihre Sprache ist gnadenlos und unwissend zynisch, was u.a. in dem Gespräch mit dem Arzt zu sehen ist:

Arzt: Fühlst du dich traurig?

Dora: Ich fühle mich immer traurig. Außer beim Ficken.

Arzt: Denkst du manchmal ans Kind?

Dora. Weg ist weg. (Bärfuss 2015: 105)

Dass Dora diese direkte stilistisch naturalistische Frechheit in den Mund gelegt wird, verbirgt aber eine tiefere Bedeutung, zumal sie offen über ihre Gefühle spricht sowie darüber, was andere gern verbergen würden. Dies verschärft ebenso die Position, die der Autor wohl auch selber vertritt, und zwar dass manche Themen, wären es Sex, Sucht oder Verbrechen, grundsätzlich gerne tabuisiert werden. Dadurch dass sie - hier am Beispiel der Sexualität - aufgegriffen werden, kann ein kleiner Beitrag dazu geleistet werden, um sie zu enttabuisieren, oder zumindest zur Diskussion zu stellen, was hingegen sehr reflexiv und zurückhaltend erfolgt, ohne zu kategorisieren oder zu verurteilen.

Bei Bärfuss steht die gesellschaftliche Kritik nicht im Vordergrund, aber er problematisiert in seinen Werken, auch in Die sexuellen Neurosen unserer Eltern die so oft gesuchten Lücken, mit denen man sich Debatten zu ersparen hofft. Der Schweizer Autor besteht aus vielerlei Zweifeln, zeigt ein Dilemma, aber keine Lösungen, was er auch selber zu bestätigen scheint: „Es wird nämlich versucht, ein Problem zu lösen, für das es keine Lösung gibt. Unser Leben endet in einer Katastrophe, und jeder Versuch, das abzumildern, mündet automatisch in Verzweiflung" (Scherer). Dorothee Hammerstein schreibt dazu:

${ }^{11}$ Andererseits sieht man aber ein, dass er die Abtreibung heuchlerisch zulässt und sie durchführt. 
Lukas Bärfuss schildert Doras Verhältnisse sachlich knapp, in Szenen, deren lakonische Würze verrät, wie viel Mühe auf sie verwendet wurde. Er enthält sich jeder Erörterung des gesellschaftlichen heißen Eisens, das man aus dieser Geschichte auch schmieden könnte. Er bezieht nicht Stellung und nimmt nicht Partei. Er beobachtet und zeigt. Zwar beobachtet er mit dem leicht boshaften Blick des Satirikers für Leute in der Bredouille, das heißt, seine Szenen nehmen immer den direktesten Weg hin zum springenden Punkt, aber er klagt nicht an. Da ist nirgends Platz für erhebendes Gerede um den heißen Brei herum. Und diese Zurückhaltung macht die Stärke seines Stücks aus. Nächst Dora natürlich. Aus ihr macht er keine Heldin und keine Heilige, und schon gar nicht ein jämmerliches Opfer. Er drängt sich nicht besserwissend vor sie. Er betrachtet sie aus fast scheuer Distanz. Er ist ganz präzis, wo es um die Details ihres Verhaltens geht, aber er überwältigt sie nicht durch auktoriales Besserwissen. So bleibt das Geheimnis ihrer Einfalt, das auch das Geheimnis ihrer Unzerstörbarkeit ist, ungelüftet. Und das ist nicht nur gut so. Das ist schön. (Hammerstein)

Die sexuellen Neurosen unserer Eltern von Lukas Bärfuss lenken die Aufmerksamkeit nicht nur auf die Tabuisierung der Sexualität von Behinderten, was am Beispiel Doras gezeigt wird, sondern auch auf die im Titel erwähnten Generationenneurosen, die sowohl Eltern als auch Groß- und Großgroßeltern betreffen, welche sich angesammelt haben und von Generation zu Generation übertragen wurden, in Form von Konventionen und allgemein geltenden „Wahrheiten“. Dabei geht es also grundsätzlich nicht um die Frage nach dem Ergebnis bestimmter gesellschaftlicher Verhaltensmuster, sondern nach deren Gründen, die wegen der Tabuisierung zu unterschiedlichen Arten von Neurosen führen. Der harte Sex, für den sich Dora so gern entscheidet, symbolisiert in dieser Härte vor allem die mentale Enge der Menschen, die von Tabus umgeben, sich keine unangenehmen Fragen stellen wollen. Bärfuss kreuze hier, wie Simone Kaempf betont, Generationenkonflikte mit ideologiebehafteten Diskursen und verhandele im Nahbereich menschlicher Beziehungen, was sich im Theater deutlicher erfahren lässt. ${ }^{12}$ Deutlicher erfahren lässt sich dies auch in dramentheoretischer Hinsicht, die solche enttabuisierenden Praktiken mit der Postmoderne und ihrer Einstellung dem (Kunst) Werk gegenüber verbindet.

\section{Postdramatisches Schreiben?}

Die sexuellen Neurosen unserer Eltern, die so frei über Sexuelles und seine Tabuisierung sprechen, sind ein gutes Beispiel für die postdramatische Auseinandersetzung mit der Gegenwart, zumal dieses Werk sich aus theoretischer Perspektive nicht mehr als traditionelles Drama betrachten lässt. Die Postmoderne, die den Text nicht mehr als Schöpfung eines genialen Subjekts, sondern als Kreuzungspunkt sieht, an dem sich eine Vielfalt von Texten und Textbezügen überlagert,

${ }^{12}$ Vgl. Simone Kaempf: Seit die Wunder abhanden kamen. In: TAZ vom 13.09.2005. http:// www.taz.de/!546619/ (Zugriff am 23.05.2017). 
ermöglicht eine offene Stellung und kreatives Ineinandergehen von Künsten, was ebenso nicht als vollendetes Ergebnis, sondern als Versuchsanordnung verstanden werden kann. Dass also heutzutage auf der Bühne alles aufgeführt wird, z.B.: engagierte Lyrik, politische Prosa, geschichtlicher Roman, usw., wundert in diesem Kontext überhaupt nicht. Gerda Poschmann, die den Begriff der ,nicht mehr dramatischen" Theatertexte geprägt hat, behauptet, dass dadurch die dramatische Form sehr unterschiedlich genutzt werden kann, z.B.: problemlos als Spiegel der Gegenwart, kritisch im Metadrama, im epischen und absurden Theater oder auch in Monodramen. Sie schreibt in ihrer Studie mit dem Titel Der nicht mehr dramatische Theatertext, dass die Entfernung, sogar Ablösung des Theaters von dem Drama, seit Jahren nicht nur eine Tatsache ist, sondern auch fortschreitet und sich weiter vertieft:

zunehmend sperren sich zeitgenössische Theaterstücke gegen eine Behandlung als Dramen im herkömmlichen Sinne, sind als solche sinnvollerweise, so scheint es, nicht inszenierbar und entziehen sich auch dem Zugriff einer Dramenanalyse, die versucht, diese Texte mithilfe traditioneller dramaturgischer Begriffe zu untersuchen. (Poschmann: 1)

Die Vorstellung von dem Drama, das bei Aristoteles einerseits als Sprachund Kunstwerk, andererseits als Spieltext und Spielvorlage betrachtet wird, d.h. mit dem Tragödientext „höher bewertet wird als die Visualität der Aufführung“ (Bayerndörfer: 2), scheint demnach völlig obsolet geworden zu sein. Das veränderte Verhältnis von Theater, Text und Drama und die Einstellung der Theatralität gegenüber hat man vor allem mit der Zeit der Moderne gründlicher zu analysieren versucht, erstens mit Edward Georg Craig und mit dem von ihm vorgeschlagenen Theater ohne dramatischen Vorgabetext, zweitens mit den wissenschaftlichen Erwägungen von Peter Szondi, der sich 1957 zu der Entwicklung des modernen Dramas von 1880 bis 1950 ausführlich geäußert hat und der als wissenschaftlicher Bahnbrecher bei den Analysen der dramatischen Form gilt. Szondi behauptete u.a., dass die sozialen und kulturellen Entwicklungen der modernen Welt das Drama so sehr umgeformt haben, dass es neu definiert werden muss, vor allem aus dem Grund, dass die traditionelle Dialogik kommunikativ nicht mehr tragfähig und daher durch andere literarische Methoden zu ersetzen ist (Szondi: 9). Wir lesen bei ihm zudem von der Verbannung all epischer Formen, die zur Krise geführt haben soll:

Seit Aristoteles haben die Theoretiker der dramatischen Dichtung das Auftreten epischer Züge in diesem Bereich an den Pranger gestellt. Wer aber heute die Entwicklung der neueren Dramatik darzustellen versucht, kann sich zu solchem Richteramt nicht mehr berufen fühlen [...]. (ebd.)

Mit Szondi wurde es klar, dass das Theater nicht unbedingt auf dramatische Texte zurückgehen muss (u.a. am Beispiel des absurden Theaters von Pirandello, des epischen Theaters von Brecht, des politischen Revues von Piscator oder der expressionistischen Ich-Dramatik). Es wurde aber auch deutlich, dass er seine the- 
oretischen Ausführungen als Rettungsversuch des Dramas verstanden hat, ${ }^{13}$ während die späteren Untersuchungen, vor allem von Hans-Peter Bayerndöfer oder Hans-Thies Lehmann etwas anderes festzustellen versuchten. Erstens entbehre das Drama schon lange seiner ästhetischen Wahrheit (Bayerndörfer: 3), zweitens umfasse das Theater zwischen den 1970er und 1990er Jahren, das als postmodern bezeichnet wird, u.a. das Theater der Dekonstruktion, plurimediales Theater, restaurativ traditionalistisches/konventionelles Theater, Theater der Gesten und Bewegungen. Dies ,schließt also die Gegenwart/die Wiederaufnahme/das Wiederwirken älterer Ästhetiken ein, auch solcher, die schon früher der dramatischen Idee auf der Ebene des Textes oder des Theaters den Abschied gegeben haben“" (Lehmann 1999: 31). Postdramatisches Theater, das sich als Arbeitsformel für Text und Bühne etabliert hat, kann leer, übervoll, nihilistisch und grotesk sein. Es zeigt einerseits fehlende Entfaltung einer Fabel oder Handlung und ist demnach eine nach Botho Strauß genannte „Involvierung von inneren und äußeren Zuständen“ (ebd. 113). Andererseits erlaubt es auch die erwähnten, älteren Ästhetiken, wodurch eine Vielfalt entsteht, die in ihrer Breite, Anerkennung und Ablehnung bestimmter Formen nur in der Postmoderne möglich ist.

Wichtig und prägend sind in diesem Diskurs auch die Thesen, die Roland Barthes aufgestellt hat, und zwar, dass jeder Text seine Wirklichkeit erreicht, indem seine Sprache befragt wird, sowie die von Jacques Derrida, der jeden Text, der für ihn aus einer unbegrenzten Zahl von Differenzen und Spuren von anderen Aussagen besteht, ${ }^{14}$ dekonstruieren ließ. Im Theater geht es aber nicht nur um die Zeichen der Sprache, sondern auch darum, dass „der Theatertext [...] den gleichen Gesetzen und Verwerfungen wie die visuellen, auditiven, gestischen, architektonischen usw. Zeichen des Theaters [unterliegt]“ (Lehmann 1999: 13). In dieser Reflexion kann man also über postdramatische Erscheinungen sprechen, zumal es sich in den Theatertexten von heute nicht mehr und nicht nur um sprachliches Gebilde handelt. Die Verbindung zwischen Theater und Text in dem Wort „Theatertext" kann dabei mehr auf das Theater als auf den Text verweisen, der nur eine mögliche Form der szenischen Gestaltung präsentiert.

Das, was Lukas Bärfuss in seinen thematisch provokanten Theatertexten vornimmt, scheint einerseits dem Brechtschen epischen Theater sehr zu ähneln - als dessen deutliche Modifizierung, andererseits aber auch der Derridischen Dekonstruktion. Mit Brecht hat er sicherlich den Verfremdungseffekt gemeinsam, mit Themen, die verblüffen und die mit ihrem Ende offen bleiben, um den Zuschauer selber zu Schlussfolgerungen zu bewegen. Seine Werke sind aber zugleich vielmehr gegenwartsbezogener als die Stücke von Brecht - auch in naturalistischer Intention - wo das Milieu in seiner Eigenmacht über den Menschen erscheint. Der Komponist und Regisseur Heiner Goebels meint in diesem Zusammenhang,

${ }^{13}$ Er dachte dabei, dass das epische Theater zum Universalschlüssel der neuen Entwicklung werden sollte.

${ }^{14}$ Dadurch also nicht ausgedacht wird, sondern wiederholt und zitiert. 
dass ,vieles von dem, was das postdramatische Theater in den achtziger und neunziger Jahren entwickelt hat, [...] letztlich nichts anderes als das [ist], was Brecht theoretisch schon vorformuliert hatte" (Goebells: 124). Die epischen Mittel sind demnach nicht nur im Postdrama verbreitet. Distanzierende Illusionsbrüche und Episierungen prägen auch fabelgestützte, realistische Dramaturgien, vor allem dort, wo eine neue politische und soziale Dramatik angestrebt wird. Hier wie in postdramatischen Experimenten begegnen narrative Vermittlungsinstanzen, ausgreifende Erzählpassagen, metatheatrale Elemente und intermediale Verfahren. Diese episierenden Mittel haben sich jedoch verselbständigt und stehen nicht mehr im Zusammenhang eines epischen Theaters im Sinne Brechts. Vielmehr „treiben“ sie, wie es Hans-Thies Lehmann bemerkt, „als Stückchen auf dem mainstream heutiger Theaterpraxis ebenso wie in den Nebengewässern des experimentellen Theaters" (Lehmann 2012: 222).

Bärfuss arbeitet in seinen Theatertexten aber auch mit Elementen des offenen, zersplitterten Dramas, er versucht die Splitter miteinander zu verbinden, so dass, wie Peter Michalzik schreibt, eine „Bewegung der Schließung“ (Michalzik: 40) entsteht, die aber dann, was hier behauptet wird, nur eine Bewegung bleibt, zumal Mythen, von denen Bärfuss selber spricht, keine vollständige Wirklichkeit herstellen können und zumal Ganzes nicht existiert, so dass man dann nur vom „begrenzten Ganzen“ sprechen kann. Michalzik schreibt hierzu über dramatische Dekonstruktionen bei Bärfuss: ,[...] traditionelle Sichtweisen kommen vor, aber sie sind entwertet in Hinsicht auf ihre Verbindlichkeit, sie sind Material einer eigenen Bewegung geworden, die ihren Standort selbst bestimmt, Teil eines Spiels, das seine Grundlagen miteinbezieht" (ebd. 41).

In seiner Art, zu schreiben, ist der Schweizer Autor vielschichtig. Man sieht, dass seine Theatertexte „ganz klassisch im Bau“ (Dössel), „linear in der Struktur und bezwingend einfach in der Sprache" (ebd.) sind, (Thomas Bühler schreibt dabei über „Textgestaltung auf der Basis der Selbstäußerung personaller Rollen und [über] den Text als zeitgenössische Spielvorlage mit klassischer Trennung zwischen Spielern und Zuschauern und einer Darstellung mittels Narration“" (Bühler: 50), man merkt aber auch, dass das Traditionelle (in der Form) hier mit dem Postdramatischen (in der von Lehmann genannten „Koexistenz divergierender Theaterkonzepte" (Lehmann 2012: 18) bei den neuesten Theatertexten)) im Einklang steht. Selbst der Autor scheint dies zu bekräftigen, wenn er behauptet, dass ein Werk für die Bühne grundsätzlich nicht vollendet ist: „Er [der Text - J.K.] verlangt nach einer Umsetzung. [...] Damit die Umsetzung gelingt, muss man in der Beschreibung einerseits sehr genau sein, andererseits aber Zwischenräume lassen“ (Gradwohl). Und diese Zwischenräume machen bei ihm ebenso das vielseitige und vielfältige Postdramatische aus.

Der Kritiker Tan Wälchli schreibt Bärfuss eine besondere Rolle zu. Er meint nämlich, dass der Autor sich von der Sackgasse, in die das postmoderne Theater gerade geraten ist, am besten zu befreien wisse. Er schreibt: „Wie das postmoderne 
Theater sich aus seiner Sackgasse zu befreien hat, weiß heute vermutlich niemand so genau wie Lukas Bärfuss“ (Wiesel: 102). Man fragt sich aber dabei, ob diese Prognose angesichts der Vielfalt und Mannigfaltigkeit der Postdramatik zutreffend ist. Eher ist es eine Verbindung des Alten mit dem Neuen, der Gestus von Offenheit und Neugier, was zeitlich und räumlich eingeschränkt bleibt, was aber im Denken nicht mehr traditionell, sondern postdramatisch ist und sich in Die sexuellen Neurosen unserer Eltern exemplarisch realisieren lässt. Nicht ohne Grund endet das Stück mit den Worten „Fin de la bobine“, dem Aufruf der Kinovorführer, die eine neue Filmrolle einlegen wollen. Das Filmische mischt sich hier mit dem Theatralischen, aber gesprengte Formen sind dabei etwas, was konstitutiv bleibt und wovor man bei Bärfuss keine Angst haben muss.

In einem Gespräch mit Iwona Uberman hat der Schweizer Autor auch noch auf etwas hingedeutet, was in dem postdramatischen Diskurs von Bedeutung sein könnte. Es handelt sich nämlich um den hier schon erwähnten Mythos, an den er glaubt und der seine Geschichten erzählen lässt:

Der Wille zu den Geschichten folgt aus dem Glauben an den Mythos, glaube ich. Ich möchte eigentlich die Mythen weitererzählen. Wichtig am Mythos scheint mir die Unverträglichkeit der Persönlichkeit mit der Geschichte, die sie erlebt. [...] Der Mythos, eigentlich jede Geschichte, kümmert sich nicht um seine Protagonisten. Er vollzieht sich, er schert sich keinen Deut um Charaktere, um die Idee der Individualität. (Uberman: 3)

Der Mythos und der Plot, die Aristoteles als Seele der Tragödie angesehen hat (mit einem kunstvoll konstruierten Handlungsverlauf), wird bei Bärfuss aber in einer anderen, dekonstruierten Form aufgenommen, die jenen Mythos wiederholt und als Fragment einer als Ganzheit unerreichbaren Wirklichkeit darlegt.

In diesem Zusammenhang lässt sich wohl behaupten, dass der Schweizer Autor seinen Text als einen zum Nachdenken bewegenden und provozierenden Beitrag zur Diskussion über Behinderungen und Sexualität verstanden wissen will. Als Beitrag, der - im Nebeneinander verschiedener Formen, zwischen traditionellen literarischen Dramen und den „nicht mehr dramatischen“ Theatertexten verankert ist - doch aber dabei als neue Dramatik, die Traditionelles und traditionelle, asketische Narrationen verwendet, die aber das Postmoderne mit dem postmodernen Denken und Bewusstsein der Aufsplitterung, der fehlenden Einheit sowie eines Kernes in dieser Abhandlung in den Vordergrund stellt - als zwischenräumliche Theatralik.

\section{Schlusswort}

In der deutschsprachigen Literatur der Schweiz zählt Lukas Bärfuss sicherlich zu den wichtigsten Vertretern des neuen Theaters und der neuen Perspektive, die dann jedoch mit den aufgenommenen Themen nicht typisch für die Schweiz wird. 
Das Schweizerische, das den Autor geprägt hat, spielt natürlich eine Rolle, im Sinne seiner Herkunft und der mentalen Öffnung in der Schweiz selbst, die um die Jahrtausendwende erfolgte, es ist aber nicht das A und O seiner künstlerischen Erwägungen, zumal sie, was am Anfang angeführt wurde, mehr global als lokal sind. Die von Bärfuss vertretene Haltung zu Theatertexten scheint dies deutlich zu bekräftigen, weil seine provokanten Themen ein breiteres Publikum finden, als dieses aus der Schweiz nur. Die Auseinandersetzung mit dem Thema „Sexualität“ und ihrer Tabuisierung bietet ebenso eine globale Diskussion an, die im europäischen Kulturraum überall stattfinden kann.

Der Schweizer Autor, der sich einfachen Stücken zuwendet (er sagt selber dazu: „Nur die kleinen Probleme sind kompliziert, die großen sind schrecklich einfach. Was ist kompliziert am Tod? Nichts, und trotzdem ist er unlösbar. Das interessiert mich. Deshalb möchte ich einfache Stücke schreiben, einfach und unlösbar" (Bärfuss; Gerstenberg; Kampert: 57)), ist in dieser Einfachheit der Postdramatik und ihren zwischenräumlichen Ebenen zuzuschreiben, von denen er sich nicht, was manche behaupten, ${ }^{15}$ abgrenzt, sondern sie zulässt und auf eine für ihn spezifische Art und Weise umsetzt. Er stellt Fragen nach dem „Selbstbestimmungsrecht von Personen, die häufig unreflektiert als schutzbedürftig angesehen werden" (Ingelmann), führt eindrucksvoll vor, was geschieht, wenn man mit diesen Fragen falsch umgeht, sie schlecht beantwortet oder gleich völlig ignoriert, (ebd.) und versucht wohl, die Sexualität junger Menschen in der Pubertät zu veranschaulichen und sie mit einer geistigen Behinderung zu verbinden, so dass der Sex als Thema letztendlich enttabuisiert werden kann - um die Gesellschaft zu berühren, zugleich aber auch gegen vereinfachte, oberflächliche Denkweisen zu wirken, erzählt durch den Mythos, der bestimmte Verhaltensmuster wiederholt und bearbeitet.

Giery Cavelty schreibt über Bärfuss' Stücke, dass sie auf keinen Fall ,trockenes Thesentheater“ (Cavelty) sind, denn „zu menschlich schillern die Protagonisten: In Bärfuss' Blick auf die Figuren verbinden sich Scharfsinnigkeit und Nachsichtigkeit zu einer Comédie humaine“" (ebd.). Und der Autor ergänzt es noch ausdrücklich selber: „Für mich ist der Mensch dort am meisten Mensch, wo er mit seinen Widersprüchen lebt und diese auszuhalten versucht. [...] Helden sind wir alle auf die gleiche Art. Schwach sein und scheitern dagegen, das ist etwas sehr Persönliches und Liebenswürdiges."16

15 z.B. von Christine Bähr, die zeigen will, dass sich der Autor von der postdramatischen Ästhetik abgrenzt. Vgl. dazu Bähr (2012: 386).

16 Ebd. 


\section{Literatur}

\section{Primärliteratur}

Bärfuss, Lukas (2015): Die sexuellen Neurosen unserer Eltern. In: Meienbergs Tod. Die sexuellen Neurosen unserer Eltern. Der Bus. Zürich.

\section{Sekundärliteratur}

Bähr, Christine (2012): Der flexible Mensch auf der Bühne. Sozialdramatik und Zeitdiagnose im Theater der Jahrtausendwende. Bielefeld.

Bärfuss, Lukas / Gerstenberg, Judith / Kampert, Britta: Tragödie der Eindeutigkeit. Lukas Bärfuss über sein Stück „Die Probe (Der brave Simon Korach) “. In: Programmheft 168. Burgtheater Wien. Spielzeit 2007/2008. S. 52-58.

Bayerndörfer, Hans Peter (2007): Vom Drama zum Theatertext? Unmaßgebliches zur Einführung. In: Christopher Balme / Hans-Peter Bayerndörfer / Dieter Borchmeyer / Andreas Höfele (Hrsg.): Studien zur Geschichte und Theorie der dramatischen Künste. Bd. 52. Tübingen.

Bucheli, Roman: Das Dilemma der guten Absicht. In: Neue Zürcher Zeitung vom 12.04.2008. http:// www.nzz.ch/das-dilemma-der-guten-absicht-1.708257 (Zugriff am 15.07.2015).

Bühler, Thomas (2007): Die Praxis schlägt zurück. Alices Reise in die Schweiz. Szenen aus dem Leben des Sterbehelfers Gustav Strom von Lukas Bärfuss. In: Balme, Christopher / Bayerndörfer, Hans-Peter / Borchmeyer, Dieter / Höfele, Andreas (Hrsg.): Studien zur Geschichte und Theorie der dramatischen Künste. Bd. 52. Tübingen. S. 43-51.

Cavelty, Gieri: Erfahrungen haltbar machen. In: Neue Zürcher Zeitung vom 20.06.2005 https:// www.nzz.ch/articleCV4WB-1.151787 (Zugriff am 15.04.2017).

Dössel, Christine: Leben ist Glaubenssache. Stephan Kimmig inszeniert „,Der Bus “ von Lukas Bärfuss am Thalia Theater in Hamburg. In: Süddeutsche Zeitung vom 1.02.2015. S. 14.

Gespräch mit Lukas Bärfus. In: http://www.teatr-pismo.pl/ludzie/650/filozof_i_poeta._rozmowa_z_ lukasem_bärfussem (Zugriff am 2.02.2014). Hervorhebung - J.K.

Goebells, Heiner (2007): Von der Unabhängigkeit der Mittel. In: Frank-M. Raddatz (Hrsg.): Brecht frisst Brecht. Berlin.

Hammerstein, Dorothee (2003): Programmheft Mülheimer Theatertage.

Ingelmann, Julian: Die Macht des Einzelnen. In: Litlog. Göttinger eMagazin für Literatur-KulturWissenschaft vom 11.08.2015. http://www.litlog.de/die-macht-des-einzelnen/ (Zugriff am 28.02.2016).

Kaempf, Simone: Seit die Wunder abhanden kamen. In: TAZ vom 13.09.2005. http://www.taz. de/!546619/ (Zugriff am 23.05.2017).

Lehmann, Hans-Thies (1999): Postdramatisches Theater. Frankfurt am Main.

Lehmann, Hans-Thies (2012): Das politische Schreiben. Essays zu Theatertexten. In: Theater der Zeit.

Lukas Bärfuss im Interview mit Noëmi Gradwohl. In: Berner Zeitung vom 3.02.2005. S. 27.

Michalzik, Peter (2008): Traditionelle neue Dramatik bei Rinke, von Mayenburg, Schimmelpfennig und Bärfuss. In: Stefan, Tigges (Hrsg.): Dramatische Transformationen. Zu gegenwärtigen Schreib- und Aufführungsstrategien im deutschsprachigen Theater. Bielefeld. S. 31-42.

Poschmann, Gerda (1997): Der nicht mehr dramatische Theatertext. Aktuelle Bühnenstücke und ihre dramaturgische Analyse. Tübingen.

Germanica Wratislaviensia 143, 2018

(C) for this edition by CNS 
Roth, Gerhard (2003): Fühlen, Denken, Handeln. Wie das Gehirn unser Verhalten steuert. Frankfurt am Main.

Scherer, Benedikt: »Ich werde immer langsamer«. Schweizer Dramatiker Lukas Bärfuss ist in Basel wie Hamburg ein gefragter Autor. In: Aargauer Zeitung/MLZ vom 4.03.2005. S. 14.

Szondi, Peter (1965): Theorie des modernen Dramas. Frankfurt am Main.

Iwona Uberman im Gespräch mit Lukas Bärfuss. In: Teatr. Nr. 11/2013. S. 3.

Weber, Ulrich / Burkard, Philipp (2002): Die neue Entspanntheit. Reto Sorg w rozmowie o wspótczesnej niemieckojęzycznej literaturze Szwajcarii. In: CH-Lit. Mitteilungen zur deutschsprachigen Literatur der Schweiz. Nr. 9.

Wiesel, Jörg (2007): Theater/Prekarität. In: Alexandra Kleihues: Figurationen, gender, literatur, kultur VIII.1, ,, armut/poverty“. Berlin. S. 95-106.

http://tls.theaterwissenschaft.ch/wiki/Lukas_Bärfuss (Zugriff am 14.07.2015).

http://www.400asa.ch/400asa/geschichte.php (Zugriff am 14.07.2015).

http://www.400asa.ch/theater/archiv/itnacht/bekenntnis99.pdf (Zugriff am 14.07.2015).

http://www.literaturfestival.com/archiv/teilnehmer/autoren/2014/lukas-baerfuss (Zugriff am 20.05.2017).

http://www.munzinger.de/search/go/document.jsp?id=00000025289 (Zugriff am 14.07.2015). 